\title{
Relação entre acuidade visual e atividades instrumentais de vida diária em idosos em seguimento ambulatorial
}

\section{Relationship between visual acuity and instrumental activities of daily living of the elderly in clinical segment}

Sheila de Melo Borges ${ }^{1}$, Fernanda Aparecida Cintra ${ }^{2}$

\section{$\underline{\text { RESUMO }}$}

Objetivo Avaliar a influêrcia da acuidade visual nas atividades instrumentais de vida diária em idosos em seguimento ambulatorial. Métodos Foram entrevistados, por meio de instrumento próprio, 64 idosos em seguimento ambulatorial num hospital universitário de Campinas/SP. Os sujeitos foram divididos em dois grupos, conforme os valores obtidos na medida da AV: o grupo I, composto pelos idosos com visão normal ou próxima do normal; e o grupo II, formado por aqueles com baixa visão. Foram realizadas correlações bivariadas entre as variáveis, a fim de identificar as seguintes relações entre os grupos de acuidade visual e as atividades instrumentais de vida diária. Resultados Foram observadas diferenças significativas entre os idosos com visão normal ou próxima do normal (grupo I) e aqueles com baixa visão (grupo II), com respeito às variáveis. O grupo II apresentou maior dificuldade do que o grupo I no escore total do instrumento, bem como nas seguintes tarefas: uso do telefone, preparo de refeições, trabalhos manuais, lavar e passar roupa e tomar corretamente os remédios. Conclusão: Neste estudo, idosos com baixa visão de ambos os sexos apresentam maior dificuldade para realizar as AIVDs do que idosos com visão normal ou próxima do normal.

Descritores: Acuidade visual; Atividades cotidianas; Autonomia pessoal; Envelhecimento; Idoso

\footnotetext{
${ }^{1}$ Mestre em Gerontologia pela Faculdade de Educação, Universidade Estadual de Campinas - UNICAMP - Campinas (SP), Brasil; ${ }^{2}$ Doutora, Professora do Departamento de Enfermagem da Universidade Estadual de Campinas - UNICAMP - Campinas (SP), Brasil; 


\section{INTRODUÇÃO}

$\mathbf{O}$ declínio biológico é inevitável durante o processo de envelhecimento, uma vez que todos os sistemas do corpo sofrem perdas tanto nos aspectos estruturais como funcionais ${ }^{(1)}$. Com a função visual não é diferente. Vários estudos populacionais internacionais relacionados aos problemas oftalmológicos no idoso se destacam em publicações nessa área: Beaver Dam Eye Study, Australian Blue Mountain Eye Study, The Blue Mountains Eye Study, Melbourne Visual Impairment Project, National Diet and Nutrition Wide Samples of British e Project SEE. Alguns destes estudos fornecem dados sobre a prevalência e a severidade da perda da visão em todo o mundo ${ }^{(2-7)}$. Estes dados mostram um aumento significativo na prevalência da deficiência visual com o aumento da idade, especialmente naqueles com 75 anos ou mais. Entre os idosos, a deficiência visual e a cegueira podem ser avaliadas como um importante problema de saúde pelo aumento de sua prevalência(8).

Dentre as perdas sensoriais, alterações na visão têm sido associada à incapacidade física ${ }^{(9)} \mathrm{e}$ corresponde à terceira causa de incapacidade funcional nos idosos, depois dos problemas relacionados ao aparelho locomotor e os de origem cardiovascular $^{(10)}$.

A visão tem um importante papel para a independência funcional, tendo em vista que mecanismos sensoriais, principalmente os visuais, resultam em declínio na habilidade para desempenhar tarefas rotineiras essenciais para os indivíduos viverem diariamente ${ }^{(11)}$.

Admite-se que a deficiência visual torna-se mais prevalente com o aumento da idade e pode interferir no desempenho tanto nas atividades básicas de vida diárias (ABVDs) quanto nas Atividades instrumentais de vida diária (AIVDs) ${ }^{(12-16)}$.

Embora a relação entre função visual e capacidade funcional relacionada às atividades de vida diária na velhice seja conhecida como indicador importante da qualidade de vida nesta população( ${ }^{(9)}$, a avaliação desta relação ainda é pouco realizada. Assim, justifica-se a realização deste estudo em idosos em seguimento ambulatorial especializado no Brasil.

Frente ao exposto, o nosso objetivo é avaliar a influência da visão, por meio da avaliação da acuidade visual, na capacidade funcional direcionada à realização das atividades instrumentais de vida diária em idosos em seguimento ambulatorial.

\section{Métodos}

\section{Delineamento do estudo}

Trata-se de um estudo transversal integrante do Projeto Temático intitulado "Qualidade de vida em idosos fragilizados: indicadores de saúde e de bem-estar subjetivo", o qual tem por objetivo geral identificar e analisar os fatores de predição e discriminação de fragilidade e bem-estar subjetivo em idosos residentes no município de Campinas e região, considerando o impacto destas variáveis sobre a qualidade de vida destas pessoas. Este Projeto é coordenado por docentes do Curso de Pós-Graduação em Gerontologia da Faculdade de Educação da Universidade Estadual de Campinas (UNICAMP), Campinas, São Paulo.

\section{Local}

O estudo tem como campo de pesquisa o Ambulatório de Geriatria do Hospital das Clínicas da UNICAMP. Esta unidade recebe pacientes encaminhados de unidades básicas de saúde e de outras especialidades médicas do próprio serviço, em sua grande maioria.

\section{População e Amostra}

Os sujeitos do Projeto Temático são idosos com 65 anos ou mais, de ambos os sexos, que vivem na comunidade e são portadores de doenças somáticas. A amostra do Projeto Temático contou com 102 sujeitos selecionados por estratos, de acordo com sua presença no respectivo ambulatório. Os dados foram coletados no período de outubro de 2005 a julho de 2006. Os critérios para a participação deste estudo foram: Inclusão: aceite em participar da pesquisa com a assinatura do Termo de Consentimento Livre e Esclarecido; Exclusão: presença de déficit cognitivo que dificultasse a compreensão e resposta do instrumento; diagnóstico de doenças neurológicas (ex: acidente vascular encefálico, doença de Parkinson, lesão medular e poliomielite) que pudessem modificar a funcionalidade do indivíduo devido à sequela da doença.

Participaram neste estudo 64 idosos com idade entre 65 e 92 anos [Média=76,33 $( \pm 6,86)]$. Predominou o sexo feminino $(56,25 \%)$ e a cor branca $(82,81 \%)$; em menor proporção encontravam-se os pardos $(10,94 \%) \mathrm{e}$ negros $(6,25 \%)$. Com respeito ao estado conjugal, $50 \%$ dos idosos eram casados, $42,19 \%$ viúvos, $3,13 \%$ divorciados e 4,69\% solteiros. Grande parcela dos idosos frequentou apenas o ensino fundamental (62,50\%), 9,38\% o ensino médio, e uma proporção significativa (28,13\%) revelou nenhuma escolaridade. A renda familiar men- 
sal informada variou entre zero e $\mathrm{R} \$ 1.200,00$ (mediana $=\mathrm{R} \$ 300,00$ reais - Salário mínimo vigente na época igual a $\mathrm{R} \$ 350,00)$.

\section{Desenvolvimento da pesquisa}

A coleta de dados do Projeto Temático foi efetuada por uma equipe treinada, da qual participou a autora do presente estudo. Nesta coleta foi utilizado um instrumento elaborado para este Projeto, que contém perguntas fechadas e escalas de avaliação. Este instrumento está dividido nas seguintes seções: I. Identificação pessoal; II. Dados sociodemográficos; III. Saúde física; IV. Antropometria; V. Mobilidade e flexibilidade;VI. Hábitos de vida; VII. Atividade física; VIII. Saúde bucal; IX. Aspectos funcionais e de satisfação com a alimentação; X. Quedas; XI. Estado cognitivo; XII. Fluência verbal; XIII. Estado depressivo; XIV. Bem-estar subjetivo; XV. Medida de independência funcional; XVI. Atividades instrumentais de vida diária; XVII. Atividades expandidas de vida diária.

As entrevistas foram individuais, realizadas em sessão única e em ambiente reservado, no dia da primeira consulta ou do retorno dos idosos no referido serviço de atendimento ambulatorial.

Para o presente estudo, foram utilizados os seguintes dados das seções:

a) Seção I: Número de identificação do sujeito;

b) Seção II: Dados sociodemográficos: gênero, idade, cor, estado conjugal, escolaridade e rendimento;

c) Seção III: Medida de acuidade visual (AV) obtida pela Tabela de Snellen;

d) Seção XVI: Atividades Instrumentais de Vida Diária de Lawton (AIVDs) ${ }^{(17)}$.

Todos os dados foram extraídos do banco de dados do Projeto Temático.

Os participantes deste estudo $(n=64)$ foram divididos em dois grupos, conforme os valores obtidos da AV:

Grupo I: $\mathrm{AV}^{3}$ 20/60 em ambos os olhos (AO) ou $\mathrm{AV}^{3}$ 20/60 no melhor olho, com correção óptica se fizer uso; fizer uso.

Grupo II: AV < 20/60 AO, com correção óptica se

Para facilitar a análise e a discussão consideramos a AV do grupo I como "normal" ou "próxima do normal" e a do grupo II como "baixa visão".

$\mathrm{Na}$ escala da AIVDs, foi utilizada a escala de Lawton e Brody (1969)(17) onde a pontuação varia entre nove e 27 pontos, o que corresponde a dependência máxima e independência máxima, respectivamente. Para cada questão são atribuídos três pontos para a tarefa realizada de forma "independente", dois pontos para

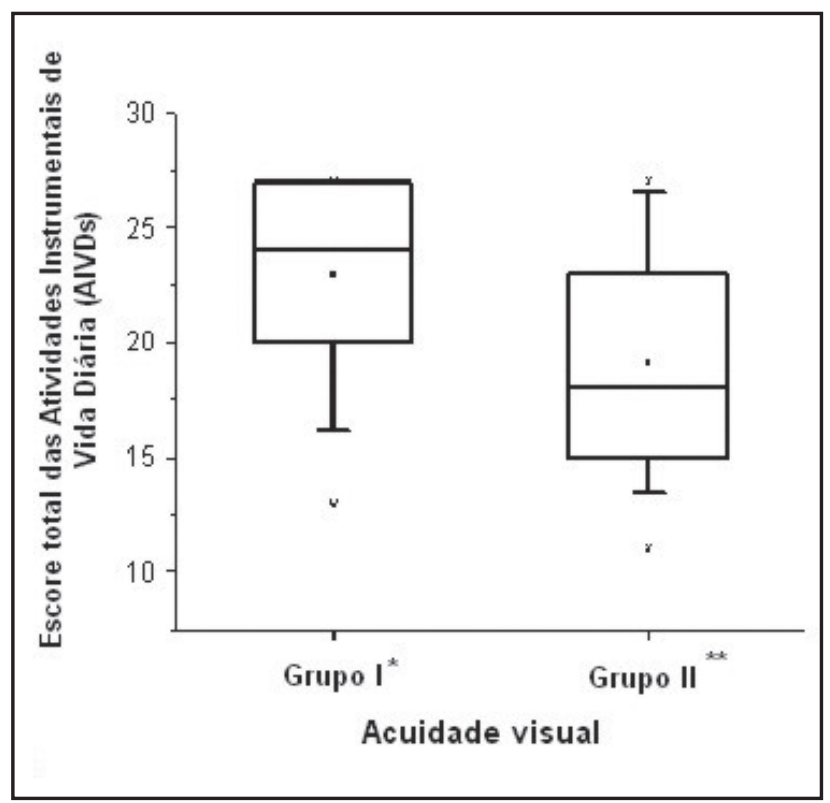

Figura 1: Relação entre acuidade visual e atividades instrumentais de vida diária

"dependência parcial" e um ponto para a "dependência total". Nesse trabalho, avaliamos a pontuação obtida em cada tarefa e o escore total do instrumento.

\section{Análise dos dados}

Os dados coletados foram inseridos no programa estatístico SPSS versão 8.0 System for Windowns e realizadas uma análises de comparação, para comparar as variáveis numéricas entre os grupos foram utilizados os testes de Mann-Whitney (2 grupos).

\section{Aspectos Éticos}

O Projeto Temático no qual este estudo está integrado foi aprovado pelo Comitê de Ética da Faculdade de Ciências Médicas da UNICAMP, parecer n 240/2003. Os pacientes foram orientados sobre o anonimato e a liberdade em desistir a qualquer momento da pesquisa ao assinarem o Termo de Consentimento Livre e Esclarecido, conforme Resolução 196/96 do Conselho Nacional de Saúde.

\section{Resultados}

Dentre os 64 idosos estudados, o grupo I foi representado por $64 \%(n=41)$, enquanto o grupo II foi representado por $36 \%(n=23)$ desta amostra.

Com respeito às AIVDs, verificamos correlação estatisticamente significativa $(\mathrm{p}=0,003)$ entre os grupos I e II e o escore total do instrumento, bem como nas 
Tabela 1

Comparação entre as variáveis numéricas dos grupos de acuidade visual e as atividades instrumentais de vida diária $(n=64)$

\begin{tabular}{|c|c|c|c|c|c|c|c|c|c|c|}
\hline $\begin{array}{l}\text { Tarefas } \\
\text { das AIVDS }\end{array}$ & Média & $\begin{array}{l}\text { Gru } \\
\text { Desvio } \\
\text { padrão }\end{array}$ & $\begin{array}{l}\text { po I } * \\
\text { Mediana }\end{array}$ & $\begin{array}{c}\text { Variação } \\
\text { observada }\end{array}$ & Média & $\begin{array}{l}\text { Grup } \\
\text { Desvio } \\
\text { padrão }\end{array}$ & $\begin{array}{l}\text { oo II ** } \\
\text { Mediana }\end{array}$ & $\begin{array}{c}\text { Variação } \\
\text { observada }\end{array}$ & $\begin{array}{r}\text { Variação } \\
\text { possível }\end{array}$ & $\begin{array}{c}* * * \text { Valor } \\
\text { de p }\end{array}$ \\
\hline Uso do telefone & 2,76 & 0,54 & 3 & 1 a 3 & $\underline{\mathbf{2}, 30}$ & 0,82 & 3 & 1 a 3 & 1 a 3 & $\underline{0,013}$ \\
\hline \multicolumn{11}{|l|}{ Deslocamento para } \\
\hline planejamento especial & 2,37 & 0,70 & 2 & 1 a 3 & 2,09 & 0,67 & 2 & 1 a 3 & 1 a 3 & 0,105 \\
\hline Fazer compras & 2,39 & 0,74 & 3 & 1 a 3 & 2,17 & 0,83 & 2 & 1 a 3 & 1 a 3 & 0,317 \\
\hline Preparar refeições & 2,80 & 0,51 & 3 & 1 a 3 & $\underline{2,26}$ & 0,92 & 3 & 1 a 3 & 1 a 3 & $\underline{0,007}$ \\
\hline Arrumar a casa & 2,46 & 0,78 & 3 & 1 a 3 & 2,13 & 0,87 & 2 & 1 a 3 & 1 a 3 & 0,116 \\
\hline Trabalhos manuais & 2,59 & 0,74 & 3 & 1 a 3 & $\underline{1,91}$ & 0,90 & 2 & 1 a 3 & 1 a 3 & $\underline{\mathbf{0 , 0 0 2}}$ \\
\hline Lavar e passar roupa & 2,39 & 0,89 & 3 & 1 a 3 & $\overline{\mathbf{1 , 7 0}}$ & 0,88 & 1 & 1 a 3 & 1 a 3 & $\underline{\mathbf{0 , 0 0 4}}$ \\
\hline \multicolumn{11}{|l|}{ Tomar remédios } \\
\hline Cuidar das finanças & 2,59 & 0,71 & 3 & 1 a 3 & 2,35 & 0,83 & 3 & 1 a 3 & 1 a 3 & 0,234 \\
\hline Escore total & 22,90 & 4,12 & 24 & 13 a 27 & $\underline{19,13}$ & 4,72 & 18 & 11 a 27 & 9 a 27 & $\underline{\mathbf{0 , 0 0 3}}$ \\
\hline
\end{tabular}

(*) Grupo I: visão normal ou próxima do normal

(**) Grupo II: baixa visão

(***) Valor de p - referente ao teste de Mann-Whitney

seguintes tarefas: uso do telefone $(\mathrm{p}=0,013)$, preparo de refeições $(p=0,007)$, trabalhos manuais $(p=0,002)$, lavar e passar roupa $(p=0,004)$ e tomar corretamente os remédios $\mathrm{p}=0,021)$. Em todas as tarefas, as médias e medianas do grupo II foram inferiores às do grupo I, o que representa maior limitação dos idosos com baixa visão para o desempenho das AIVDs (tabela 1).

Na figura 1, observamos diferença entre os grupos de AV em relação às AIVDs. Os grupos são diferentes quando observamos as caixas em relação às suas características: maior dispersão na caixa do grupo II, observação discrepante em ambos os grupos (o grupo I mostra observações menores e no grupo II, verificamos observações menores e maiores que as demais dentro do grupo). As medianas e as caudas não se sobrepõem, o que revela diferença entre os dois grupos. O teste de Mann-Whitney revelou diferença significante entre os dois grupos $(p=0,003)$.

\section{DiscussÃo}

Fisiologicamente no envelhecimento, existe uma relação hierárquica de perdas de habilidades funcionais, na qual observa-se inicialmente a perda das habilidades para as AIVDs e posteriormente para as ABVDs ${ }^{(18,19)}$. Isto ocorre devido à diferença de complexidade entre tais atividades: as AIVDs são, na maioria das vezes, mais complexas do que as ABVDs ${ }^{(20)}$. As AIVDs englobam as atividades funcionais necessárias para um indivíduo vi- ver de forma independente na comunidade, na qual a presença de dificuldades implica mais em uma redistribuição de tarefas entre os membros familiares (como alguém para ir fazer compras ou auxiliar nas tarefas domésticas) e menos em cuidado direto ${ }^{(19)}$.

Os idosos que reportam mais dificuldade nas tarefas de ABVDs e AIVDs têm duas vezes mais chances de baixa visão ${ }^{(13)}$. Outro estudo, de um grupo de pesquisadores da Finlândia, observou que pessoas com diminuição da AV são cinco vezes mais prováveis a apresentar dificuldade em AIVDs do que uma pessoa com boa $\mathrm{AV}^{(16)}$. No nosso estudo, observamos uma correlação de perdas das AIVDs em idosos com baixa visão, onde os idosos do grupo II (baixa visão) apresentaram mais dificuldade em realizar as AIVDs do que os idosos do grupo I (visão normal ou próxima do normal). Apesar de idosos de uma forma geral serem mais propensos a apresentarem algum comprometimento em AIVDs, apenas os idosos do grupo de baixa visão apresentaram comprometimento em algumas tarefas das AIVDs.

Observamos, também no nosso estudo, que os idosos com baixa visão apresentaram dificuldade em utilizar o telefone, preparar refeições, trabalhos manuais, lavar e passar roupa e tomar corretamente os remédios.

A função visual mostra estreita relação com a mobilidade ${ }^{(16)}$, pois durante a locomoção, a visão é necessária para monitorar e analisar a localização e o movimento do corpo, bem como as condições ambientais para as quais o sistema motor deveria res- 
ponder ${ }^{(21)}$. Porém, o declínio visual tem sido associado não apenas com a diminuição da mobilidade, mas também com a necessidade de fazer compras em mercados, preparo de refeições, andar fora de casa, cuidar das finanças ${ }^{(22)}$, bem como tomar a medicação corretamente ${ }^{(23)}$. Esta associação nem sempre é linear e facilmente compreendida, podendo essa relação entre deficiências sensoriais e dependência nas AIVDs ser indireta e complexa ${ }^{(24)}$. Idosos que relatam ter dificuldade em três ou mais atividades de AIVDs são mais sugestionadas a ter incapacidades graves do que aqueles que relatam dificuldade em uma ou duas tarefas ${ }^{(13)}$. Portanto, os idosos do grupo de baixa visão do nosso estudo, segundo a literatura, está mais propenso à incapacidade grave, já que em geral, eles apresentam dificuldade em cinco atividades das AIVDs.

De qualquer forma, idosos de ambos os sexos, que apresentam dificuldades no desempenho das AIVDs, em geral necessitam de auxílio de outras pessoas que atuem como elo de ligação entre eles e seu entorno social, contribuindo muitas vezes para o afastamento do idoso do convívio social e, consequentemente, a tendência ao isolamento ${ }^{(19)}$.

\section{CONCLUSÃO}

Observamos em nosso estudo que idosos com baixa visão de ambos os sexos apresentam maior dificuldade para realizar as AIVDs do que idosos com visão normal ou próxima do normal.

\section{Agradecimentos}

Ao curso de Pós-graduação em Gerontologia da UNICAMP, as professoras Fernanda Aparecida Cintra e Anita Liberalesso Neri, aos colegas de coleta de dados do Projeto temático e aos idosos que colaboraram com a pesquisa.

\section{AbStract}

Objective: To evaluate the influence of visual acuity in instrumental activities of daily living in elderly patients in follow-up period. Methods: through own instruments, sixty-four elderly men and women were interviewed in clinical segment at an academic hospital of Campinas/ SP. They were then divided into two groups in accordance to the values that were obtained through the measure of the VA: the first group consisted of elderly people with normal vision or close to the normal, and the second group was formed of those with low vision. Bivariate correlations were made between the variables in order to identify the following relations: between groups of visual and instrumental activities of daily living. Results: Significant differences between the elderly with normal vision or close to the normal (group I) and those with low vision (group II) were found: the group II had greater difficulty than the group I total score of the instrument and the following tasks: telephone use, meal preparation, handicrafts, launder and take the medicine correctly. Conclusion: This study, elderly people with low vision of both sexes have more difficulty to carry out AIVDs than elderly people with normal vision or close to normal.

Keywords: Visual acuity; Activities of daily living; Personal autonomy; Aging; Aged

\section{RefERÊNCIAS}

1. Ribeiro JE, Freitas MM; Araújo GS, Rocha TH. Associação entre aspectos depressivo e déficit visual causado por catarata em pacientes idosos. Arq Bras Oftalmol. 2004; 67(5):795-9.

2. Attebo K, Mitchell P, Smith W. Visual acuity and the causes of visual loss in Australia. The Blue Mountains Eye Study. Ophthalmology. 1996;103(3):357-364.

3. Klaver CC, Wolfs RC, Vingerling JR, Hofman A, De Jong PT. Age-specific prevalence and causes of blindness and visual impairment in an older population. The Rotterdan Study. Arch Ophthalmol. 1998; 116(5):653-8.

4. van der Pols JC, Bates CJ, McGraw PV, Thompson JR, Reacher M, Prentice A, Finch S. Visual acuity measurements in a national sample of Britsh elderly people. Br J Ophthalmol. 2000; 84(2):165- 70.

5. Weih LM, VanNewkirk MR, McCarty CA, Taylor HR. Agespecific causes of bilateral visual impairment. Arch Ophthalmol. 2000; 118(2):653-8.

6. Klein R, Klein BEK, Lee KE, Chappell RJ. Changes in visual acuity in a population over a 10 -year period. Ophthalmology. 2001; 108(10):1757-66.

7. Klein BEK, Moss SE, Klein R, Lee KE, Cruickshanks KJ. Associations of visual function with physical outcomes and limitations 5 years later in a older population. Ophthalmology. 2003:110(4):644-650.

8. Tsai CY, Woung LC, Chou P. Yang CS, Sheu MM, Wu JR, Chuang TL, Tung TH. The current status of visual disability in the elderly population of Tawan. Jpn J Ophthalmol.2005; 49(2)::166-72.

9. Salive ME, Guralnik J, Glynn RJ, Christen W, Wallace RB. Association of visual impairment with mobility and physical function. J Am Geriatr Soc. 1994; 42(3)::287- 92.

10. Swagerty DL Jr. The impact of age-related visual impairment on functional independence in the elderly. Kans Med. 1995; 96(10:24- 6.

11. Bergman B, Bergstrom A, Sjostrand J. Longitudinal changes in visual acuity and visual ability in a cohort followed from the age of 70 to 88 years. Acta Ophthalmol Scand. 1999; 77(3):286-92.

12. Scott IU, Schein OD, West SK, Bandeen-Roche K, Enger C, Folstein MF. Functional status and quality of life measurement among ophthalmic patients. Arch Ophthalmol. 1994; 112(3):329- 35 . 
13. West SK, Munoz B, Rubin GS, Schein OD, Bandeen-Roche K, Zeger S, German S, Fried LP. Function and visual impairment in a population-based study of older adults. The SEE project. Salisbury Eye Evaluation. Invest Ophthalmol Vis Sci. 1997;38(1):72-82.

14. Crews DE. Artificial environments and aging population: designing for age-related functional losses. J.Physiol Anthropol Appl Human Sci. 2005; 24(1):103-9.

15. Berger S, Porell F. The association between low vision and function. J Aging Health. 2008;20(5):504-25.

16. Laitinen A, Sainio P, Koskinen S, Rudanko SL, Laatikainen L, Aromaa A. The Association Between Visual Acuity and Functional Limitations: Findings from a Nationally Representative Population Survey. Ophthalmic Epidemiology. 2007;14(6):333 - 342

17. Lawton MP, Brody EM. Assessment of older people: selfmaintaining and instrumental activities of daily living. Gerontologist. 1969;9(3):179-86.

18. Ramos LR, Rosa TEC, Oliveira ZM, Medina MCG, Santos FRG. Perfil do idoso em área metropolitana na região sudeste do Brasil: resultados de inquérito domiciliar. Rev Saúde Pública. 1993;27(2): 87-94.

19. Lebrão ML, Laurenti R. Saúde, bem-estar e envelhecimento: o estudo SABE no Município de São Paulo. Rev. Bras. Epidemiol. 2005; 8,(2):127-141.
20. Teresi JA, Crass PS, Golden RR. Some applications of latent trait analysis to the measurement of ADL. J Gerontol. 1984; 44(5):196-204.

21. Sakari-Rantala R, Era P, Rantanen T, Heikkinen E. Associations of sensory-motor functions with poor mobility in 75-and 80 year-old people. Scand J Rehabil Med. 1998; 30(2):121-7.

22. Branch LG. Howowitz A, Carr C. The implications for everyday life of incident self-reported visual decline among people over age 65 living in the community. Gerontologist. 1989; 29(3):359-365.

23. Windham BG, Griswold ME, Fried LP, Rubin GS, Xue QL, Carlson MC. Impaired vision and the ability to take medications. J Am Geriatr Soc. 2006;54(5):863-5.

24. Dargent-Molina P, Hays M, Bréart G. Sensory impairments and physical disability in aged women living at home. International Journal of Epidemiology. 1996; 25(3):621-9.
Endereço para correspondência:
Sheila de Melo Borges
Rua Júlio Conceição, ${ }^{\circ} 231$
CEP 11015-540 - Santos, (SP)
Tel: (13) 32223851
Email: sheila_geronto@yahoo.com.br 